\title{
A new ordering parameter of spectral energy distributions from synchrotron-self-Compton emitting blazars
}

\author{
Reinhard Schlickeiser*† \\ Institut für Theoretische Physik, Lehrstuhl IV: Weltraum- und Astrophysik, Ruhr-Universität \\ Bochum, 44780 Bochum, Germany \\ E-mail: rschetp 4 .rub.de

\section{Michael Zacharias} \\ Institut für Theoretische Physik, Lehrstuhl IV: Weltraum- und Astrophysik, Ruhr-Universität \\ Bochum, 44780 Bochum, Germany \\ E-mail: mzatp4.rub.de
}

\begin{abstract}
The broadband SEDs of blazars exhibit two broad spectral components, which in leptonic emission models are attributed to synchrotron radiation and SSC radiation of relativistic electrons. During high state phases, the high-frequency SSC component often dominates the low-frequency synchrotron component, implying that the inverse Compton SSC losses of electrons are at least equal to or greater than the synchrotron losses of electrons. The linear synchrotron cooling, usually included in radiation models of blazars, then has to be supplemented by the SSC cooling. Here, we present an analytical solution to the kinetic equation of relativistic electrons subject to the combined synchrotron and nonlinear synchrotron self-Compton cooling for monoenergetic injection. The electron distribution functions and the time-integrated synchrotron fluences depend sensitively on an injection parameter, determined by the injection conditions of the relativistic electrons in the emission region.
\end{abstract}

25th Texas Symposium on Relativistic Astrophysics

December 6-10, 2010

Heidelberg, Germany

\footnotetext{
* Speaker.

${ }^{\dagger}$ We acknowledge support from the German Ministry for Education and Research (BMBF) through Verbundforschung Astroteilchenphysik grant 05A08PC1 and the Deutsche Forschungsgemeinschaft through grants Schl 201/201 and Schl 201/23-1.
} 


\section{Introduction}

The broadband continuum spectra of blazars are dominated by nonthermal emission and often consist of two distinct broad components. In leptonic emission models (for review see Böttcher [1]) synchrotron radiation from highly relativistic electrons generates the low-energy component whereas the high-energy component results from Compton interactions of the same relativistic electron population. In many blazars the Compton emission component dominates the synchrotron emission component. Here we investigate the radiative signatures of the injection and subsequent, self-consistent synchrotron and SSC cooling of monoenergetic electrons, including the case of SSC dominated radiative output.

The dominance of the SSC component over the synchrotron component in this case implies that the inverse Compton SSC losses of electrons are at least equal or greater than the synchrotron losses of electrons, even more when the intergalactic deabsorption of the $\mathrm{TeV}$ emission from the cosmic infrared background is accounted for. The ratio of the observed SSC to synchrotron photon luminosity from the same population of electrons $n(\gamma)$

$$
\frac{L_{S S C}^{*}}{L_{s y}^{*}}=\frac{\int d V \int_{1}^{\infty} d \gamma n(\gamma)\left|\gamma_{S S C}\right|}{\int d V \int_{1}^{\infty} d \gamma n(\gamma)\left|\dot{\gamma}_{S}\right|}
$$

directly reflects the ratio of the corresponding loss rates, because of the identical Doppler boosting factors[2] of synchrotron and SSC emission.

In the case of the dominance of the first-order SSC component over the synchrotron component, Schlickeiser[3] (hereafter referrred to as paper S) has pointed out that then the linear synchrotron cooling rate, included standardly in radiation models of blazars, has to be replaced by the nonlinear SSC cooling rate. In the Thomson limit (hereafter referred to as SST-losses) the SST energy loss rate of a single electron

$$
|\dot{\gamma}|_{\text {SST }} \simeq A_{0} \gamma^{2} \int_{0}^{\infty} d \tilde{\gamma} \tilde{\gamma}^{2} n(\tilde{\gamma}, t), A_{0}=\frac{3 \sigma_{T} c_{1} P_{0} R \varepsilon_{0}^{2}}{m c^{2}}
$$

depends on the energy integral of the actual electron spectrum $n(\gamma, t)$, reflecting that the energy integral determines the number density of the target synchrotron photons in the source. The dependence on the energy integral is a collective effect completely different from the linear synchrotron energy loss rate of a single electron in a constant magnetic field

$$
|\dot{\gamma}|_{S}=D_{0} \gamma^{2}, \quad D_{0}=\frac{4}{3} \frac{c \sigma_{T}}{m c^{2}} U_{B}=1.29 \cdot 10^{-9} b^{2} \mathrm{~s}^{-1},
$$

which is solely determined by the magnetic field strength $B=b$ Gauss and the electron Lorentz factor $\gamma$. The notation in Eqs. (1.2) and (1.3) is the same as in paper $\mathrm{S}: P_{0}=3.2 \cdot 10^{12} \mathrm{eV}^{-1} \mathrm{~s}^{-1}$, $\varepsilon_{0}=1.16 \cdot 10^{-8} \mathrm{beV}, R$ is the radius of the spherical source, $c$ denotes the speed of light, $\sigma_{T}=$ $6.65 \cdot 10^{-25} \mathrm{~cm}^{2}$ is the Thomson cross section and $c_{1}=0.684$.

\section{Solution of electron kinetic equation for combined synchrotron and SST cooling}

The competition between the instanteneous injection of ultrarelativistic electrons $(\gamma \gg 1)$ with the arbitrary injection distribution function $q(\gamma)$ at time $t=0$ and the combined synchrotron and SST radiative losses 


$$
\frac{d \gamma}{d t}=\dot{\gamma}=\dot{\gamma}_{S}+\dot{\gamma}_{\mathrm{SST}}=-\gamma^{2}\left(D_{0}+A_{0} \int_{0}^{\infty} d \gamma \gamma^{2} n(\gamma, t)\right)
$$

is described by the time-dependent kinetic equation for the volume-averaged relativistic electron population inside the radiating source[4]:

$$
\frac{\partial n(\gamma, t)}{\partial t}-\frac{\partial}{\partial \gamma}\left[\gamma^{2}\left(D_{0}+A_{0} \int_{0}^{\infty} d \gamma \gamma^{2} n(\gamma, t)\right) n(\gamma, t)\right]=q(\gamma) \delta(t)
$$

where $n(\gamma, t)$ denotes the volume-averaged differential electron number density. Introducing the implicit time variable $T(t)$ by

$$
\frac{d T}{d t}=D_{0}+A_{0} \int_{1}^{\infty} d \gamma \gamma^{2} n(\gamma, t)=D_{0}+A_{0} \int_{0}^{\infty} d \gamma R(\gamma, t)
$$

where $R(\gamma, t)=\gamma^{2} n(\gamma, t)$, yields for the kinetic equation (2.2)

$$
\frac{\partial R}{\partial T}-\gamma^{2} \frac{\partial R}{\partial \gamma}=\gamma^{2} q(\gamma) \delta(T)
$$

With the boundary condition $R(\gamma=\infty, T)=0$ its solution by the method of characteristics is (with the step function $H$ )

$$
\gamma^{2} n=R(\gamma, T)=\int_{\gamma}^{\infty} d \gamma^{\prime} q\left(\gamma^{\prime}\right) \delta\left(\frac{1}{\gamma^{\prime}}-\frac{1}{\gamma}+T\right)=H[T] H[1-T \gamma]\left(\frac{\gamma}{1-\gamma T}\right)^{2} q\left(\frac{\gamma}{1-\gamma T}\right)
$$

Inserting this solution we obtain for Eq. (2.3)

$$
\frac{d T}{d t}=D_{0}+A_{0} H[T] \int_{0}^{1 / T} d \gamma\left(\frac{\gamma}{1-\gamma T}\right)^{2} q\left(\frac{\gamma}{1-\gamma T}\right)
$$

yielding upon integration the time-dependence of implicit time scale $T(t)$ for specified injection distributions.

\subsection{Monoenergetic injection}

Nearly monoenergetic electron injection distributions result from the pile-up mechanism[5, 6, 7], i.e. the simultaneous operation of first-order Fermi acceleration and radiative losses of electrons. For the injection of monoenergetic distributions $q(\gamma)=Q_{0} \delta\left(\gamma-\gamma_{0}\right)$ with the total volume-averaged density of injected electrons $Q_{0}=3 N /\left(4 \pi R^{3}\right)=2.39 \cdot 10^{4} N_{50} R_{15}^{-3} \mathrm{~cm}^{-3}$, where we scale the total number of injected electrons $N=10^{50} N_{50}$ and the source size $R=10^{15} R_{15} \mathrm{~cm}$, we obtain for Eq. (2.6)

$$
\frac{1}{D_{0}} \frac{d T}{d t}=1+\frac{1}{\gamma_{B}^{2}\left(T+\gamma_{0}^{-1}\right)^{2}}
$$

The characteristic electron Lorentz factor in Eq. (2.7)

$$
\gamma_{B}=\left(\frac{D_{0}}{A_{0} Q_{0}}\right)^{1 / 2}=\frac{217 R_{15}}{N_{50}^{1 / 2}}
$$


reflects the injection conditions of the relativistic electrons. Obviously, the more compact the source is, and the more electrons are injected, the smaller the electron injection Lorentz factor $\gamma_{B}$ is. Eq. (2.7) is solved by

$$
\frac{1+T \gamma_{0}}{\alpha}-\arctan \left(\frac{1+T \gamma_{0}}{\alpha}\right)=\frac{D_{0} \gamma_{0} t}{\alpha}+c_{0}
$$

with the integration constant $c_{0}$ and the injection parameter

$$
\alpha=\frac{\gamma_{0}}{\gamma_{B}}=46 \frac{\gamma_{4} N_{50}^{1 / 2}}{R_{15}},
$$

where we scale $\gamma_{0}=10^{4} \gamma_{4}$. Unfortunately, Eq. (2.9) cannot be inverted to a closed form giving the dependence $T(t)$, which is needed in Eq. (2.5) to determine the electron distribution function as a function of $\gamma$ and $t$. However, an approximate but reasonably accurate inversion is possible by using the asymptotic expansions of the arctan-function for small and large values of the injection parameter $\alpha$ compared to unity.

\subsection{Small injection parameter}

For small values of $\alpha \ll 1$ we approximate Eq. (2.9) with $T(t=0)=0$ as $T \simeq D_{0} t$. According to Eq. (2.5) the time evolution of the electron distribution function is solely determined by the linear synchrotron losses, and is given by

$$
n(\gamma, t)=Q_{0} H\left[\gamma_{0}-\gamma\right] \delta\left(\gamma-\frac{\gamma_{0}}{1+D_{0} \gamma_{0} t}\right)
$$

\subsection{Large injection parameter}

For large injection parameters $\alpha \gg 1$ and early times $0 \leq T \leq T_{c}=(\alpha-1) / \gamma_{0}$, corresponding to

$$
0 \leq t \leq t_{c}=\frac{\alpha^{3}-1}{3 \gamma_{B} D_{0} \alpha^{3}} \simeq \frac{1}{3 \gamma_{B} D_{0}}=\frac{1.2 \cdot 10^{6} N_{50}^{1 / 2}}{R_{15} b^{2}} \mathrm{~s}
$$

we approximate Eq. (2.9) as

$$
D_{0} t \simeq \frac{1}{3 \gamma_{0} \alpha^{2}}\left[\left(1+T \gamma_{0}\right)^{3}-1\right]
$$

yielding according to Eq. (2.5)

$$
\begin{aligned}
& n\left(\gamma, \gamma_{0}, t \leq t_{c}\right)=\frac{Q_{0} H\left[\gamma_{0}-\gamma\right] \delta\left(\frac{\gamma}{1-\gamma T}-\gamma_{0}\right)}{(1-\gamma T)^{2}}=Q_{0} H\left[\gamma_{0}-\gamma\right] \delta\left(\gamma-\frac{\gamma_{0}}{1+\gamma_{0} T}\right)=Q_{0} H\left[\gamma_{0}-\gamma\right] H\left[t_{c}-t\right] \\
& \quad \times \delta\left(\gamma-\frac{\gamma_{0}}{\left(1+3 D_{0} \gamma_{0} \alpha^{2} t\right)^{1 / 3}}\right)=Q_{0} H\left[\gamma_{0}-\gamma\right] H\left[t_{c}-t\right] \delta\left(\gamma-\frac{\gamma_{0}}{\left(1+3 A_{0} Q_{0} \gamma_{0}^{3} t\right)^{1 / 3}}\right)
\end{aligned}
$$

which agrees with the nonlinear SST solution of S, his Eq. (28).

For late times $t \geq t_{c}$ Eq. (2.9) yields 


$$
T=D_{0} t+\frac{\alpha-1}{3 \alpha^{3} \gamma_{B}}\left[2 \alpha^{2}-(\alpha+1)\right]
$$

which for $t=t_{c}$ agrees with the approximation (2.13). From Eq. (2.5) we then obtain

$$
n\left(\gamma, \gamma_{0}, t \geq t_{c}\right)=Q_{0} H\left[\gamma_{B}-\gamma\right] H\left[t-t_{c}\right] \delta\left(\gamma-\frac{\gamma_{B}}{\frac{1+2 \alpha^{3}}{3 \alpha^{3}}+D_{0} \gamma_{B} t}\right)
$$

a modified linear cooling solutions. Note that both solutions (2.14) and (2.16) indicate that at time $t_{c}$ the electrons have cooled to the characteristic Lorentz factor $\gamma_{B}$.

Provided electrons are injected with Lorentz factors much higher than $\gamma_{B}$, given in Eq. (2.8), they initially cool down to the characteristic Lorentz factor $\gamma_{B}$ by nonlinear SST-cooling until time $t_{c}$. At later times they further cool to lower energies according to the modified cooling solution (2.16). If the electrons are injected with Lorentz factors much smaller than $\gamma_{B}$ they undergo only linear synchrotron cooling at all energies with no influence of the SST cooling. The characteristic Lorentz factor $\gamma_{B}$ is only determined by the injection conditions, whereas the time scale $t_{c}$ also depends on the magnetic field strength. This different cooling behaviour for large and small injection energies affects the synchrotron and SSC intensities and fluences.

\subsection{Power law injection}

Zacharias and Schlickeiser[8] find a similar behavior for initial power law injection. Any extended injection energy spectrum is immediately quenched by the intense radiation losses to a nearly monoenergetic distribution function at either the lower or upper cutoff of the injection spectrum, depending on the steepness of the injected distribution. Therefore the assumption of monoenergetic injection is very well justified.

\section{Intrinsic synchrotron radiation fluences}

With the electron distribution functions $n(\gamma, t)$ we calculate the optically thin synchrotron intensities $I(\varepsilon, t)$. In order to collect enough photons, intensities are often averaged or integrated over long enough time intervals. For rapidly varying photon intensities this corresponds to fractional fluences which are given by the time-integrated intensities. The total fluence spectra are $F(\varepsilon)=\int_{0}^{\infty} d t I(\varepsilon, t)$.

For small injection energy $\alpha \ll 1$ the total synchrotron fluence is

$$
F_{S}(\varepsilon) \simeq F_{0 S} \begin{cases}c_{0}\left(\frac{E_{0}}{\varepsilon}\right)^{1 / 2} & \text { for } \varepsilon \ll E_{0} \\ \left(\frac{E_{0}}{\varepsilon}\right) \exp \left(-\varepsilon / E_{0}\right) & \text { for } \varepsilon \gg E_{0}\end{cases}
$$

where $c_{0}=0.95302$ and $E_{0}=1.74 b \gamma_{4}^{2} \mathrm{eV}$ denotes the intial characteristic synchrotron photon energy.

For the high injection energy case $\alpha \gg 1$ the total synchrotron fluence varies as[9]

$$
F_{h}(\varepsilon) \simeq F_{0 h} \begin{cases}c_{0} \alpha^{2}\left(\frac{E_{0}}{\varepsilon}\right)^{1 / 2} & \text { for } \varepsilon \ll E_{0} / \alpha^{2} \\ c_{2}\left(\frac{E_{0}}{\varepsilon}\right)^{3 / 2} & \text { for } E_{0} / \alpha^{2} \ll \varepsilon \ll E_{0} \\ \left(\frac{E_{0}}{\varepsilon}\right) \exp \left(-\varepsilon / E_{0}\right) & \text { for } \varepsilon \gg E_{0}\end{cases}
$$


Fig. 1 shows the fluence SEDs $N(x) \propto x F(x)$ for small $\left(\alpha_{s}=0.1\right)$ and high $\left(\alpha_{h}=100\right)$ injection conditions in terms of the normalized synchrotron photon energy $x=\varepsilon / E_{0}$.

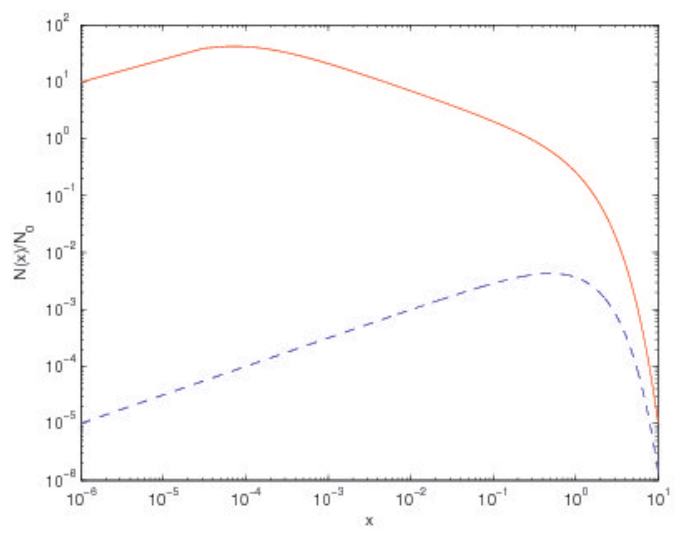

Figure 1: Total synchrotron fluence SED $N(x)$ as a function of the normalized synchrotron photon energy $x=\varepsilon / E_{0}$ for high $\left(\alpha_{h}=100\right.$, full curve) and small $\left(\alpha_{s}=0.1\right.$, dashed curve) injection conditions calculated for $\gamma_{0}=10^{4}$. From [9].

At high synchrotron photon energies $\left(\varepsilon \gg E_{0}\right)$ the total synchrotron fluences for small and high injection energy exhibit the same exponential cut-off. However, at low energies $\left(\varepsilon \ll E_{0}\right)$ we find markedly different power law behaviours for the two injection cases:

D1) In the high injection case the synchrotron SED peaks at a photon energy which is a factor $1.4 \alpha_{h}^{2}=1.4 \cdot 10^{-4}$ smaller than the peak in the small injection case.

D2) The high injection SED is a broken power law with spectral indices +0.5 below and -0.5 above the peak energy $x_{B} \ll 1$, respectively, and it cuts-off exponentially at photon energies $x>1$. Below the peak energy $x_{B}$ the time of maximum synchrotron intensity decreases as $t_{\max } \propto \varepsilon^{-1 / 2}$, whereas above the peak energy $x_{B}$ it decreases more rapidly as $t_{\max } \propto \varepsilon^{-3 / 2}$ due to the severe additional SST losses.

D3) The small injection SED is a single power law with spectral index +0.5 below the peak energy $x_{p}=0.5$, and it cuts-off exponentially at photon energies $x>1$. Here the time of maximum synchrotron intensity decreases as $t_{\max } \propto \varepsilon^{-1 / 2}$ at all energies $x<1$ because in the small injection case the SST-losses do not contribute.

\section{Summary and conclusions}

We presented the analytical solution to the synchrotron and non-linear synchrotron-self-Compton cooling in the Thomson regime (SST) of monoenergetic electrons. Based on our analytical solutions, we evaluated the time-integrated synchrotron fluences whose properties are solely determined by the injection parameter $\alpha$. We find qualitatively different results depending on whether electron cooling is initially Compton dominated (high injection energy parameter $\alpha$ ) or it is always synchrotron dominated (low $\alpha$ ). In the low- $\alpha$ case, the resulting fluence spectrum exhibits a simple exponentially cut-off power-law behaviour, $S_{V} \propto v^{1 / 2} e^{-v / v_{0}}$. In contrast, in the high- $\alpha$ case, we find a broken power-law with exponential cutoff, parametrized in the form $S_{V} \propto v^{1 / 2} \frac{v_{B}}{v+v_{B}} e^{-v / v_{0}}$. 
The analytically calculated synchrotron fluence SEDs and light curve peak times agree well with the corresponding numerically calculated quantities using the radiation code of Böttcher et al. [10]. Based on our analysis we propose the following interpretation of multiwavelength blazar SEDs:

Blazars, where the $\gamma$-ray fluence is much larger than the synchrotron fluence, are regarded as high injection energy sources. Here, the synchrotron fluence should exhibit the symmetric broken power law behaviour Eq. (3.2) around the synchrotron peak energy that is a factor $\left(\alpha_{h} \gamma_{0}\right)^{2}$ smaller than the SSC peak energy. Below and above $v_{B}$ the synchrotron light curve peak times exhibit different frequency dependences $t_{\max }\left(v<v_{B}\right) \propto v^{-1 / 2}$ and $t_{\max }\left(v>v_{b}\right) \propto v^{-3 / 2}$, respectively, resulting from the additional severe SST-losses at $v>v_{B}$.

Blazars, where the $\gamma$-ray fluence is much smaller than the synchrotron fluence, are regarded as small injection energy sources. Here, the synchrotron fluence exhibits the single power law behaviour (D3) up to a higher synchrotron peak energy that is a factor $\gamma_{0}^{2}$ smaller than the SSC peak energy. In this case the synchrotron light curve peak time exhibits the standard linear synchrotron cooling decrease $t_{\max }(v) \propto v^{-1 / 2}$ at all frequencies.

Work in progess indicates that the corresponding SSC fluences and SSC lightcurves are also solely determined by the injection parameter $\alpha$ with characteristic different spectral properties.

If the injection Lorentz factor $\gamma_{0}$ and the size of the source are the same, different values of the injection parameter $\alpha$ result from different total numbers of instantaneously injected electrons. E.g., the high injection case $\alpha_{h}=100$ results for $N_{50}=4.7$, whereas the low injection case $\alpha_{s}=0.1$ needs $N_{50}=4.7 \cdot 10^{-6}$.

\section{References}

[1] Böttcher, M., 2007, Astroph. \& Space Sci. 309, 95

[2] Dermer, C. D., Schlickeiser, R., 2002, ApJ 575, 667

[3] Schlickeiser, R., 2009, MNRAS 398, 1483 (S)

[4] Kardashev, N. S., 1962, Sov. Astron. J. 6, 317

[5] Schlickeiser, R., 1984, A\& A 136, 227

[6] Schneider. P., 1993, A\& A 278, 315

[7] Jauch, Z., Duschl, W. J., 1999, in:The Central Parsecs of the Galaxy, ASP Conference Series, Vol. 186. Ed. by H. Falcke, A. Cotera, W. J. Duschl, F. Melia, and M. J. Rieke. p. 169

[8] Zacharias, M., Schlickeiser, R., 2010, A\& A 524, A31

[9] Schlickeiser, R., Böttcher, M., Menzler, U., 2010, A\& A 519, A9

[10] Böttcher, M., Mause, H., Schlickeiser, R., 1997, A\& A 324, 395 